\title{
Neoadjuvant chemotherapy with trastuzumab in HER2-positive breast cancer: pathologic complete response rate, predictive and prognostic factors
}

\author{
I.P.C. Buzatto ${ }^{1}$, A. Ribeiro-Silva ${ }^{2}$, J.M. Andrade ${ }^{1}$, H.H.A. Carrara ${ }^{1}$, W.A. Silveira ${ }^{1}$ and D.G. Tiezzi ${ }^{1}$ \\ ${ }^{1}$ Setor de Mastologia, Departamento de Ginecologia e Obstetrícia, Faculdade de Medicina de Ribeirão Preto, \\ Universidade de São Paulo, Ribeirão Preto, SP, Brasil \\ ${ }^{2}$ Departamento de Patologia, Faculdade de Medicina de Ribeirão Preto, Universidade de São Paulo, Ribeirão Preto, SP, Brasil
}

\begin{abstract}
The purpose of this study was to retrospectively review the pathologic complete response (pCR) rate from patients ( $n=86$ ) with stage II and III HER2-positive breast cancer treated with neoadjuvant chemotherapy at our institution from 2008 to 2013 and to determine possible predictive and prognostic factors. Immunohistochemistry for hormone receptors and Ki-67 was carried out. Clinical and pathological features were analyzed as predictive factors of response to therapy. For survival analysis, we used Kaplan-Meier curves to estimate 5-year survival rates and the log-rank test to compare the curves. The addition of trastuzumab to neoadjuvant chemotherapy significantly improved pCR rate from 4.8 to $46.8 \%$, regardless of the number of preoperative trastuzumab cycles $(P=0.0012)$. Stage II patients achieved a higher response rate compared to stage III $(P=0.03)$. The diseasefree and overall survivals were not significantly different between the group of patients that received trastuzumab in the neoadjuvant setting (56.3 and $70 \%$ at 5 years, respectively) and the group that initiated it post-operatively $(75.8$ and $88.7 \%$ at 5 years, respectively). Axillary pCR post neoadjuvant chemotherapy with trastuzumab was associated with reduced risk of recurrence $(\mathrm{HR}=0.34 ; \mathrm{P}=0.03)$ and death $(\mathrm{HR}=0.21 ; \mathrm{P}=0.02)$. In conclusion, we confirmed that trastuzumab improves $\mathrm{pCR}$ rates and verified that this improvement occurs even with less than four cycles of the drug. Hormone receptors and Ki-67 expressions were not predictive of response in this subset of patients. Axillary pCR clearly denotes prognosis after neoadjuvant target therapy and should be considered to be a marker of resistance, providing an opportunity to investigate new strategies for HER2-positive treatment.
\end{abstract}

Key words: Neoadjuvant chemotherapy; Trastuzumab; Pathologic complete response; Predictive factors of response; Prognosis

\section{Introduction}

Breast cancer is the most common malignancy among women all over the world, and it is considered a heterogeneous disease. Around $25 \%$ of all breast cancer overexpress the human epidermal growth factor receptor 2 (HER2), and is traditionally associated with worse prognosis (1). The activation of HER2 occurs through dimerization with other proteins of the family, triggering multiple downstream pathways required for the abnormal proliferation of cancer cells (2).

Neoadjuvant chemotherapy is the standard of care for patients with locally advanced breast cancer. Even though it does not enhance overall survival when compared with adjuvant therapy, neoadjuvant chemotherapy has some secondary benefits (1). It can contribute to surgical downstaging and increase rates of breast-conserving surgery, in addition to providing an in vivo assessment of tumor response to chemotherapy (3).

Trastuzumab is a humanized monoclonal antibody that binds to the extracellular domain of HER2. It can potently suppress cancer cells' growth, proliferation, and survival in direct and indirect manners (2). It is well known that trastuzumab has survival benefits when associated with chemotherapy in the treatment of patients with early operable and metastatic HER2-positive breast cancer (4-6). In the neoadjuvant setting, when associated with chemotherapy, trastuzumab significantly improved pathologic complete response, and reduced the risk of relapse, disease progression and death when compared to chemotherapy alone (4). However, the available studies compared patients that received neoadjuvant chemotherapy

Correspondence: D.G. Tiezzi: <dtiezzi@usp.br>

Received July 25, 2016 | Accepted September 21, 2016 
with trastuzumab to patients that did not receive trastuzumab at all or with HER2-negative tumors $(4,7)$. It is necessary to investigate if initiating trastuzumab in the neoadjuvant setting has the same survival benefits when compared to patients that used it postoperatively.

Pathologic complete response ( $\mathrm{PCR}$ ) is usually defined as absence of residual invasive cancer in breast and lymph nodes in surgical specimens after neoadjuvant therapy. It is considered an important prognostic factor and is associated with long-term survival. pCR has been adopted as the primary end point for neoadjuvant trials and reaches up to $78 \%$ in patients treated with trastuzumab $(3,7)$. However, a significant fraction of these patients eventually relapse or develop progressive disease. This suggests that these tumors have intrinsic or acquired mechanisms of resistance to targeted therapies (8).

In this article, we reviewed the rate of $\mathrm{pCR}$ from patients with HER2-positive breast cancer treated with neoadjuvant chemotherapy at our institution and investigated possible clinical, histological and immunohistochemical predictive and prognostic factors. This knowledge would allow a better selection of patients that could benefit from therapy with trastuzumab. In addition, costs and adverse events could be minimized, leading to an individualized cancer treatment (9).

\section{Patients and Methods}

This cohort study with retrospective data collection evaluated clinical, histological, and immunohistochemical characteristics of all patients with histologically confirmed HER2-positive, stage II and III breast cancer, treated with neoadjuvant chemotherapy between 2008 and 2013 at our institution. The Institutional Review Board of the Hospital das Clínicas, Faculdade de Medicina de Ribeirão Preto, SP, Brazil, approved this study (\#497.095).

\section{Procedures}

The HER2 status was assessed by immunohistochemistry (IHC) and tumors were considered HER2-positive when $\mathrm{IHC}$ staining was $3+$ (uniform, intense membrane staining of $>30 \%$ of invasive tumor cells) and $2+$ (uniform, weak membrane staining of $>30 \%$ of invasive tumor cells), in which the HER2 gene amplification was confirmed by chromogenic in situ hybridization ( $\mathrm{CISH})$.

We used microscopically selected representative samples from breast tissues stored in our Pathology department. A paraffin tissue microarray (TMA) block was built to minimize experimental variability and reduce costs. We used the Tissue Microarray Builder Kit (Histopathology Ltd., Hungary) to arrange 24 cylinders of $2 \mathrm{~mm}^{2}$ in one recipient paraffin block.

Immunohistochemical staining for estrogen receptor, progesterone receptor and $\mathrm{Ki}-67$ was performed in the TMA sections using mouse monoclonal antibody at 1:200, 1:200 and 1:100 dilutions, respectively (NovoCastra, UK).
To quantify the expression of $\mathrm{Ki}-67$ we scanned the slides with the automated scanning system Aperio XT (Aperio Technologies, USA), and used the software ImageScope (USA) for cell count. A $14 \%$ cut-off was used to define a high expression of $\mathrm{Ki}-67$. At least 500 contiguous tumor cells were counted to establish the index.

In order to confirm the amplification of the HER2 gene we performed a dual color chromogenic $(\mathrm{CISH})$ assay using the Kit $\mathrm{CISH}^{\mathrm{TM}}$ HER2 SPOT-Light ${ }^{\mathrm{R}}$, Zymed (Invitrogen, USA) in the TMA sections. The interpretation followed the College of American Pathologists guidelines. The amplification was confirmed in all 41 tumors tested.

\section{Treatment}

Patients were treated with neoadjuvant chemotherapy with anthracyclines and/or taxanes, for at least 4 cycles, according to the current protocol. The 86 patients included in the study were divided into two groups: 65 patients received neoadjuvant chemotherapy with trastuzumab followed by adjuvant trastuzumab while 21 patients received neoadjuvant chemotherapy alone followed by adjuvant trastuzumab.

The chemotherapy regimens used were: 1) 4 cycles of $75 \mathrm{mg} / \mathrm{m}^{2}$ epirubicin + 500-600 mg/m $\mathrm{m}^{2}$ cyclophosphamide followed by 4 cycles of $100 \mathrm{mg} / \mathrm{m}^{2}$ docetaxel every 21 days +/- trastuzumab; 2) 4 to 6 cycles of $80-100 \mathrm{mg} / \mathrm{m}^{2}$ docetaxel + trastuzumab every 21 days; 3) 6 cycles of 500 $\mathrm{mg} / \mathrm{m}^{2}$ fluorouracil $+75 \mathrm{mg} / \mathrm{m}^{2}$ epirubicin $+500 \mathrm{mg} / \mathrm{m}^{2}$ cyclophosphamide every 21 days; 4) 4 cycles of $75 \mathrm{mg} / \mathrm{m}^{2}$ docetaxel $+60 \mathrm{mg} / \mathrm{m}^{2}$ epirubicin every 21 days. All patients completed 1 year of treatment with adjuvant trastuzumab, except those that developed severe toxicity.

\section{Statistical analyses}

Disease-free survival was considered as the length of time between the surgery and the first recurrence event (local or distant). Overall survival was considered as the length of time between the cancer diagnosis and death. Patients that were lost to follow-up or did not reach any event (recurrence or death) were censored in the analysis.

Statistical analyses were done with GraphPad Prism 6 software (USA). To analyze the categorical variables, we used Pearson chi-square test or Fisher's exact test. For survival analysis, we used Kaplan-Meier curves to estimate 5-year survival rates and the log-rank test to compare the curves. Statistical significance was assumed at a 0.05 level $(P<0.05)$.

\section{Results}

All of the 86 patients were treated with adjuvant trastuzumab, 66 completed 1 year of adjuvant treatment, 8 developed cardiac toxicity severe enough to interrupt the drug $(9.5 \%)$, while 11 were still under treatment with trastuzumab at the time of the study. One patient from the 
Table 1. Characteristics of the 86 patients with HER2-positive breast cancer treated with neoadjuvant chemotherapy with or without trastuzumab (T) from 2008 to 2013.

\begin{tabular}{lcccc}
\hline Characteristic & $\begin{array}{c}\text { T group } \\
(\mathrm{n}=46)\end{array}$ & $\begin{array}{c}\text { Chemo/T group } \\
(\mathrm{n}=19)\end{array}$ & $\begin{array}{c}\text { Chemo group } \\
(\mathrm{n}=21)\end{array}$ & P value \\
\hline $\begin{array}{l}\text { Mean age } \pm \text { SE (years) } \\
\text { Clinical tumor stage }{ }^{\mathrm{a}}\end{array}$ & $48.7 \pm 1.70$ & $52 \pm 1.85$ & $48.5 \pm 2.15$ & 0.4 \\
$\quad$ II & $9(20)$ & $4(21)$ & $9(43)$ & 0.1 \\
III & $37(80)$ & $15(79)$ & $12(57)$ & \\
Histological tumor grade ${ }^{\mathrm{b}}$ & & & & 0.08 \\
$\quad$ and 2 & $26(57)$ & $10(53)$ & $18(86)$ & \\
3 & $12(26)$ & $7(37)$ & $3(14)$ & \\
Not informed & $8(17)$ & $2(11)$ & $0(0)$ & 0.2 \\
Hormone receptors status ${ }^{\mathrm{c}}$ & & & & \\
$\quad$ ER and/or PR positive & $20(43)$ & $10(52)$ & $14(67)$ & $7(33)$ \\
$\quad$ ER and PR negative & $26(57)$ & $9(47)$ & $1(5)$ & $\mathbf{0 . 0 0 1}$ \\
pCR & $19(42)$ & $11(58)$ & \\
\hline
\end{tabular}

Data are reported as numbers (\%). T group: patients that received at least 4 cycles of neoadjuvant chemotherapy with trastuzumab; Chemo/T group: patients that received 1-3 cycles of neoadjuvant chemotherapy with trastuzumab; Chemo group: patients that received neoadjuvant chemotherapy without trastuzumab. ${ }^{\mathrm{a}} \mathrm{TNM}$ classification according to the International Union Against Cancer (10); ${ }^{\mathrm{b}} \mathrm{Grading}$ according to Bloom-Richardson (11) was taken from pathology reports; 'Immunohistochemistry was confirmed in samples from core-biopsy stored in our Pathology department. Values $>1 \%$ were considered positive. ${ }^{\mathrm{d}}$ One patient in the $\mathrm{T}$ group died during the neoadjuvant treatment and was excluded from the analysis. ER: estrogen receptor; PR: progesterone receptor; $\mathrm{PCR}$ : pathologic complete response. Fisher's exact test was used for statistical analyses.

first group died during the neoadjuvant treatment from febrile neutropenia and, therefore, did not receive surgical or adjuvant treatment.

The addition of trastuzumab to the neoadjuvant chemotherapy significantly improved the pCR rate. Of the 64 patients that received at least one cycle of trastuzumab in the neoadjuvant setting, 30 achieved pCR $(46.8 \%)$, while in the group of 21 patients treated with neoadjuvant chemotherapy without trastuzumab only 1 achieved pCR (4.8\%).

In a subgroup analysis, we evaluated if the number of trastuzumab cycles received preoperatively influenced the pCR rate. Of the 65 patients treated with neoadjuvant chemotherapy with trastuzumab, 46 received at least 4 cycles of trastuzumab (T group) and 19 received only 1 to 3 cycles of the drug together with neoadjuvant chemotherapy (Chemo/T group). As can be seen in Table 1, the improvement in the $\mathrm{PCR}$ rate was consistent regardless of the number of trastuzumab cycles received preoperatively. The baseline characteristics of the patients are also shown in Table 1. The groups were similar in age, clinical tumor stage at diagnosis, tumor grade and positivity to hormone receptors.

The rates of breast-conserving surgery were not significantly influenced by the addition of trastuzumab nor was pCR. Of patients treated with neoadjuvant chemotherapy with trastuzumab, 33\% had conservative surgery, compared with $52 \%$ from the chemotherapy alone $(P=0.1)$. In the group of patients that achieved pCR, 35\% underwent breastconserving surgery, while in the group with residual tumor in the breast this rate was $39 \%(P=0.8)$.

The disease-free and overall survival (Figure 1) were not significantly different between the group of patients that started trastuzumab in the neoadjuvant setting and the group treated with neoadjuvant chemotherapy alone and adjuvant trastuzumab (56.3 vs $75.8 \%$ at 5 years, $\mathrm{P}=0.38$, and 70 vs $88.7 \%$ at 5 years, $\mathrm{P}=0.26$, respectively).

We investigated clinical, histological and immunohistochemical predictive factors for response to neoadjuvant chemotherapy with trastuzumab in the 65 patients treated with at least one cycle of the drug preoperatively (Table 2). The only predictive factor for pCR in our sample was the clinical stage at diagnosis; $75 \%$ of the patients in stage II achieved pCR, compared to $40 \%$ in stage III $(P=0.03)$. Negativity to hormone receptor, histological high grade and high Ki-67 were not predictive factors for $\mathrm{pCR}$.

We evaluated possible prognostic factors in our population of HER2-positive breast cancer patients treated with neoadjuvant chemotherapy associated with trastuzumab. pCR is considered an important prognostic factor and is associated with better disease-free and overall survival (12). pCR (no invasive residual tumor in breast and axilla) was not a statistically significant prognostic factor in our study. However, pCR in the axilla (no residual tumor in the lymph nodes, regardless to the response in the breast) was an independent prognostic factor (Figure 2). 

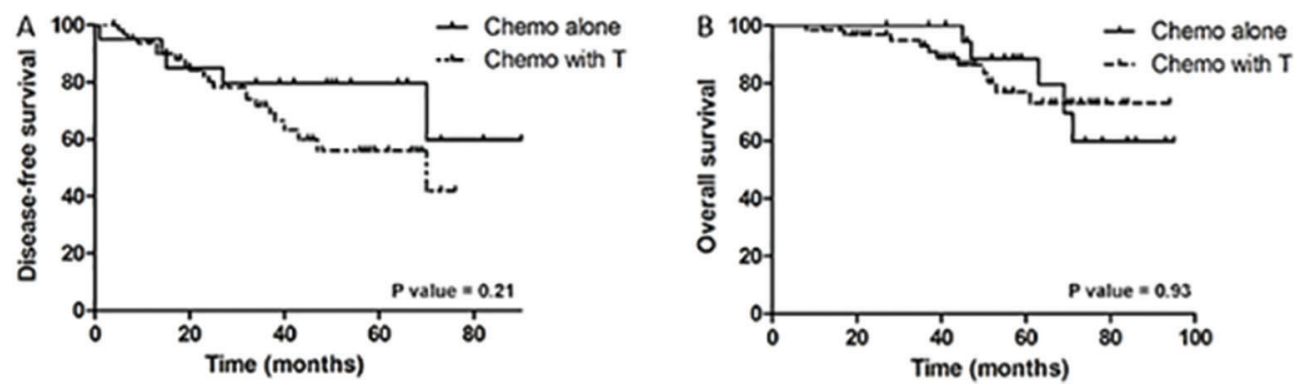

Figure 1. Disease-free $(A)$ and overall $(B)$ survival of the 86 patients with HER2-positive breast cancer treated with neoadjuvant chemotherapy (Chemo) with or without trastuzumab $(\mathrm{T})$. All patients received adjuvant treatment with trastuzumab. Log-rank test was used to compare the curves.

Table 2. Possible predictive factors of pathologic complete response ( $p C R$ ) to neoadjuvant chemotherapy with trastuzumab in HER2-positive locally advanced breast cancer patients.

\begin{tabular}{|c|c|c|c|}
\hline Possible predictive factors of response & $\mathrm{pCR}$ & No pCR & $P$ value \\
\hline Clinical stage at diagnosis ${ }^{a}$ & & & 0.03 \\
\hline II & $9(75)$ & $3(25)$ & \\
\hline III & $21(40)$ & $31(59)$ & \\
\hline Histological grading ${ }^{\mathrm{b}}$ & & & 0.8 \\
\hline Grade 2 & $16(44)$ & $20(55)$ & \\
\hline Grade 3 & $9(47)$ & $10(52)$ & \\
\hline Not informed & $5(55)$ & $4(44)$ & \\
\hline Estrogen receptor status & & & 0.7 \\
\hline ER positive & $13(44)$ & $16(55)$ & \\
\hline ER negative & $17(48)$ & $18(51)$ & \\
\hline Progesterone receptor status & & & 0.8 \\
\hline PR positive & $9(45)$ & $11(55)$ & \\
\hline PR negative & $21(47)$ & $23(52)$ & \\
\hline Ki-67 index & & & 0.6 \\
\hline$>14 \%$ (High risk of recurrence) & $19(59)$ & $13(40)$ & \\
\hline$\leqslant 14 \%$ (Low risk of recurrence) & $5(50)$ & $5(50)$ & \\
\hline Not evaluated & $6(27)$ & $16(72)$ & \\
\hline
\end{tabular}

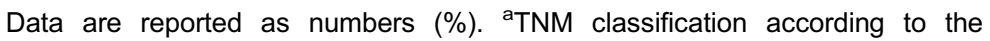
International Union Against Cancer (10); ${ }^{b}$ Grading according to BloomRichardson was taken from pathology reports (11). Statistical analysis was done with Fisher's exact test.

The 5 -year disease-free survival was $59.4 \%$ in the group of patients with axillary $\mathrm{pCR}$ and $45.7 \%$ in the group with residual burden in the axilla $(P=0.019)$. The overall survival in the group of patients with axillary pCR was $88 \%$ while in the group without axillary pCR was 55\% ( $P=0.04)$.

\section{Discussion}

Our findings confirmed that the addition of trastuzumab to neoadjuvant chemotherapy significantly improves $\mathrm{pCR}$ rates. This is consistent with other trials that demonstrated that 1 year of trastuzumab starting as neoadjuvant almost doubled pCR rates (4). The ideal number of trastuzumab cycles to be done preoperatively is not well established in the literature. A German study published in 2011 did not observe better pCR rates in patients receiving more than 4 cycles of neoadjuvant trastuzumab (13). Our study suggests that the improvement in pCR rates persists even with less than four cycles of the drug.

In a meta-analysis published in 2011, even though the probability to achieve PCR was higher in the trastuzumab plus chemotherapy group (HR 1.85; $\mathrm{P}<0.001)$, breastconserving surgery rates were similar to the group that used chemotherapy alone (3). Our study had similar findings; the 

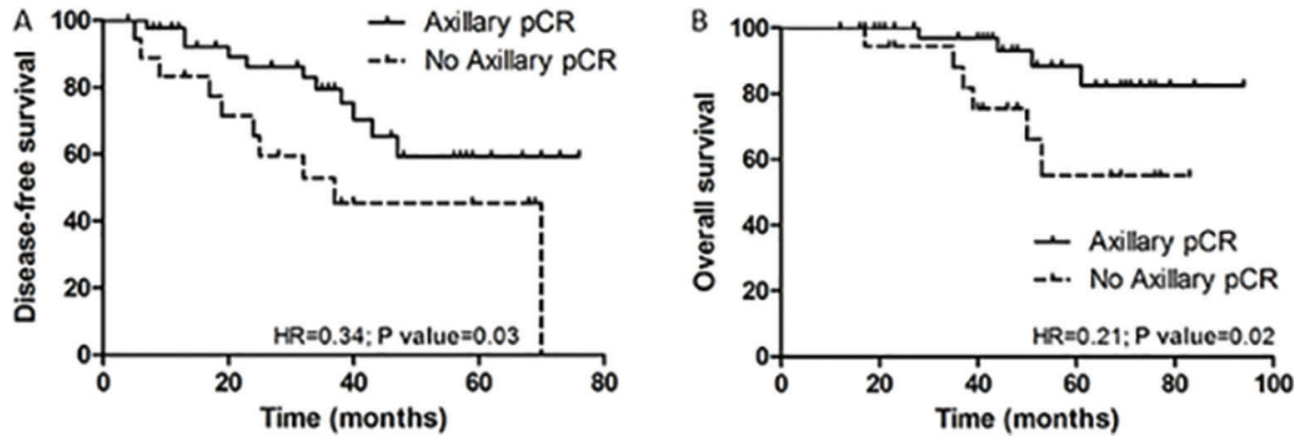

Figure 2. Disease-free $(A)$ and overall $(B)$ survival of the 63 patients with HER2-positive breast cancer treated with neoadjuvant chemotherapy with trastuzumab by axillary pathologic complete response ( $p C R)$ status. One patient died during neoadjuvant chemotherapy and was excluded from the analyses and 1 patient was excluded because the data of axillary involvement was not available. Log-rank test was used to compare the curves. HR: hazard ratio.

rates of breast conserving surgery were not influenced by the addition of trastuzumab or by pCR rates. The low rate of conservative surgery in the present study $(38 \%)$ may be a reflex of the high proportion of tumors with skin involvement (34\% of T4 tumors). The subgroup analysis with only stage II tumors found $66 \%$ of conservative surgeries.

The 3 major trials that evaluated neoadjuvant treatment with trastuzumab had a limitation in the survival analyses, because they compared it with patients that did not receive adjuvant trastuzumab and patients with HER2 negative tumors $(4,14,15)$. Our study demonstrated that neoadjuvant trastuzumab is equivalent to adjuvant trastuzumab. This result is in agreement with several trials that compared neoadjuvant to adjuvant chemotherapy; in general, the moment of administration of systemic therapy in breast cancer does not affect survival $(16,17)$.

Even though trastuzumab presented a great improvement in the treatment of HER2-positive breast cancer, it is associated with relevant adverse cardiac events and with significantly elevated cost of treatment $(9,18,19)$. In our study, $9.5 \%$ of patients had to interrupt the drug because of cardiac toxicity. This has increased the interest in identifying predictive factors of response (20). We investigated clinical, histological and immunohistochemical predictive factors of response to neoadjuvant chemotherapy with

\section{References}

1. Chang HR. Trastuzumab-based neoadjuvant therapy in patients with HER2-positive breast cancer. Cancer 2010; 116: 2856-2867, doi: 10.1002/cncr.25120.

2. Vu T, Claret FX. Trastuzumab: updated mechanisms of action and resistance in breast cancer. Front Oncol 2012; 2: 62, doi: 10.3389/fonc.2012.00062.

3. Valachis A, Mauri D, Polyzos NP, Chlouverakis G, Mavroudis D, Georgoulias V. Trastuzumab combined to neoadjuvant chemotherapy in patients with HER2-positive breast cancer: a systematic review and meta-analysis. Breast 2011; 20: 485-490, doi: 10.1016/j.breast.2011.06.009. trastuzumab and found statistical significance only for clinical stage at diagnosis.

Our findings reinforce the importance of early diagnoses in some aspects. Only 22 of the 86 patients were at stage II disease, but they had significantly greater chance of achieving $\mathrm{pCR}$ and having conservative surgery when compared to the patients at stage III. The relevance of the TNM staging has been questioned in the last few years (21). A recent study suggests that the biomarkers add important predictive and prognostic information to the TNM staging, especially in locally advanced tumors, but they do not replace it (21). Our data confirms this finding. Among the routinely used biomarkers (hormone receptor, Ki-67, histological grade), none was capable to predict pCR, except for the clinical stage at diagnosis.

We evaluated possible prognostic factors in our population of HER2-positive breast cancer patients treated with neoadjuvant chemotherapy associated with trastuzumab. Several studies have demonstrated a relation between the number of positive nodes in the axilla after neoadjuvant treatment and survival (22-25). Our data suggest that axillary $\mathrm{pCR}$ might be the most important independent prognostic factor and residual disease in the axilla should be considered a marker of resistance, providing an opportunity to investigate new strategies for HER2-positive treatment.

4. Gianni L, Eiermann W, Semiglazov V, Manikhas A, Lluch A, Tjulandin $S$, et al. Neoadjuvant chemotherapy with trastuzumab followed by adjuvant trastuzumab versus neoadjuvant chemotherapy alone, in patients with HER2-positive locally advanced breast cancer (the NOAH trial): a randomised controlled superiority trial with a parallel HER2-negative cohort. Lancet 2010; 375: 377-384, doi: 10.1016/S0140-6736(09) 61964-4.

5. Slamon DJ, Leyland-Jones B, Shak S, Fuchs H, Paton V, Bajamonde $A$, et al. Use of chemotherapy plus a monoclonal antibody against HER2 for metastatic breast cancer that 
overexpresses HER2. N Engl J Med 2001; 344: 783-792, doi: $10.1056 / N E J M 200103153441101$.

6. Smith I, Procter M, Gelber RD, Guillaume S, Feyereislova A, Dowsett $M$, et al. 2-year follow-up of trastuzumab after adjuvant chemotherapy in HER2-positive breast cancer: a randomised controlled trial. Lancet 2007; 369: 29-36, doi: 10.1016/S0140-6736(07)60028-2.

7. Symmans WF, Peintinger $F$, Hatzis $C$, Rajan $R$, Kuerer $H$, Valero $\mathrm{V}$, et al. Measurement of residual breast cancer burden to predict survival after neoadjuvant chemotherapy. J Clin Oncol 2007; 25: 4414-4422, doi: 10.1200/JCO.2007. 10.6823.

8. Rexer BN, Arteaga CL. Intrinsic and acquired resistance to HER2-targeted therapies in HER2 gene-amplified breast cancer: mechanisms and clinical implications. Crit Rev Oncog 2012; 17: 1-16, doi: 10.1615/CritRevOncog.v17.i1.20.

9. Anonymous. Breast cancer by the numbers. PT 2014; 39 : 213-214 (Abstract).

10. Sobin LH, Gospodarowicz MK, Wittekind C. The TNM classification of malignant tumours. 7th edn. city: WileyBlackwell; 2009.

11. Bloom HJ, Richardson WW. Histological grading and prognosis in breast cancer; a study of 1409 cases of which 359 have been followed for 15 years. Br J Cancer 1957; 11: 359-377, doi: 10.1038/bjc.1957.43.

12. Kuerer HM, Newman LA, Smith TL, Ames FC, Hunt KK, Dhingra $\mathrm{K}$, et al. Clinical course of breast cancer patients with complete pathologic primary tumor and axillary lymph node response to doxorubicin-based neoadjuvant chemotherapy. J Clin Oncol 1999; 17: 460-469.

13. von Minckwitz G, Untch M, Nuesch E, Loibl S, Kaufmann M, Kummel $S$, et al. Impact of treatment characteristics on response of different breast cancer phenotypes: pooled analysis of the German neo-adjuvant chemotherapy trials. Breast Cancer Res Treat 2011; 125: 145-156, doi: 10.1007/ s10549-010-1228-x.

14. Buzdar AU, Ibrahim NK, Francis D, Booser DJ, Thomas ES, Theriault RL, et al. Significantly higher pathologic complete remission rate after neoadjuvant therapy with trastuzumab, paclitaxel, and epirubicin chemotherapy: results of a randomized trial in human epidermal growth factor receptor 2-positive operable breast cancer. J Clin Oncol 2005; 23: 3676-3685, doi: 10.1200/JCO.2005.07.032.

15. Untch M, Rezai M, Loibl S, Fasching PA, Huober J, Tesch H, et al. Neoadjuvant treatment with trastuzumab in HER2-positive breast cancer: results from the GeparQuattro study. J Clin Oncol 2010; 28: 2024-2031, doi: 10.1200/JCO.2009.23.8451.
16. Mauri D, Pavlidis N, loannidis JP. Neoadjuvant versus adjuvant systemic treatment in breast cancer: a metaanalysis. J Natl Cancer Inst 2005; 97: 188-194, doi: 10.1093/ jnci/dji021.

17. Smith BL. Neoadjuvant versus adjuvant systemic therapy for operable breast cancer: equivalent outcomes? Ann Surg 2013; 257: 180-181, doi: 10.1097/SLA.0b013e318 $280 a 688$

18. Du F, Yuan P, Zhu W, Wang J, Ma F, Fan Y, et al. Is it safe to give anthracyclines concurrently with trastuzumab in neoadjuvant or metastatic settings for HER2-positive breast cancer? A meta-analysis of randomized controlled trials. Med Oncol 2014; 31: 340, doi: 10.1007/s12032-014-0340-x.

19. Xue J, Jiang Z, Qi F, Lv S, Zhang S, Wang T, et al. Risk of trastuzumab-related cardiotoxicity in early breast cancer patients: a prospective observational study. J Breast Cancer 2014; 17: 363-369, doi: 10.4048/jbc.2014.17.4.363.

20. Guiu S, Mouret Reynier MA, Toure M, Coudert B. Predictive factors of response in HER2-positive breast cancer treated by neoadjuvant therapy. J Oncol 2013; 2013: 854121, doi: $10.1155 / 2013 / 854121$.

21. Orucevic A, Chen J, McLoughlin JM, Heidel RE, Panella T, Bell J. Is the TNM staging system for breast cancer still relevant in the era of biomarkers and emerging personalized medicine for breast cancer - an institution's 10-year experience. Breast J 2015; 21: 147-154, doi: 10.1111/ tbj.12367.

22. Zhang GC, Zhang YF, Xu FP, Qian XK, Guo ZB, Ren CY, et al. Axillary lymph node status, adjusted for pathologic complete response in breast and axilla after neoadjuvant chemotherapy, predicts differential disease-free survival in breast cancer. Curr Oncol 2013; 20: e180-e192, doi: 10.3747/co.20.1294.

23. Botti C, Vici P, Lopez M, Scinto AF, Cognetti F, Cavaliere R. Prognostic value of lymph node metastases after neoadjuvant chemotherapy for large-sized operable carcinoma of the breast. J Am Coll Surg 1995; 181: 202-208.

24. Keam B, Im SA, Kim HJ, Oh DY, Kim JH, Lee SH, et al. Clinical significance of axillary nodal ratio in stage II/III breast cancer treated with neoadjuvant chemotherapy. Breast Cancer Res Treat 2009; 116: 153-160, doi: 10.1007/s10549-0080160-9.

25. Cure H, Amat S, Penault-Llorca F, le Bouedec G, Ferriere JP, Mouret-Reynier MA, et al. Prognostic value of residual node involvement in operable breast cancer after induction chemotherapy. Breast Cancer Res Treat 2002; 76: 37-45, doi: 10.1023/A:1020274709327. 